\title{
Impossibility of Exact Flipping of Three Arbitrary Quantum States Via Incomparability
}

\author{
Indrani Chattopadhyay* and Debasis Sarkar ${ }^{\dagger}$ \\ July 3, 2018 \\ Department of Applied Mathematics, University of Calcutta, \\ 92, A.P.C. Road, Kolkata- 700009, India.
}

\begin{abstract}
We present here a scheme that relates seemingly two different kinds of physical impossibilities of quantum information processing. We derive, exact flipping of three arbitrary states not lying in one great circle is not possible with certainty, by using the existence of incomparable states which are not interconvertible deterministically by local operations and classical communications. In contrast, considering the non-existence of exact universal flipper, the incomparability of a pair of bipartite pure entangled states can be established.

PACS number(s): 03.67.Hk, 03.65.Ud.
\end{abstract}

The linear superposition principle of quantum mechanical amplitudes give it such a strange structure that some operations which are obvious in classical domain are found to be impossible in the quantum arena. Some well known impossibilities are, no-cloning [1, no-deleting [2, no-flipping [3, etc., 4. Efforts are made to find inter-relations between those impossibilities and the basic dynamics of quantum mechanics. For example, the no-cloning principle directly follows from the linearity and unitary dynamics of the quantum mechanical systems [5]. In spite of the non-local character of quantum mechanics [6], it cannot be used to send signals faster than light. We find

*ichattopadhyay@yahoo.co.in

†dsappmath@caluniv.ac.in, debasis1x@yahoo.co.in 
a peaceful coexistence between no-signalling condition and the no-go principles, like, no-cloning, no-deleting, probabilistic cloning, no-flipping, etc., [7. 8].

The structure of the allowed operations performed on the quantum systems shared between some spatially separated parties imposes some restrictions on the systems. Allowing local operations along with classical communications (in short, LOCC) between separated parties, one could find some impossibilities, like the principle of 'Non-increase of entanglement by LOCC'. This has been connected with no-cloning, no-deleting [9] and further with the no-flipping principle 8]. In a quite similar manner we could find another impossibility from the transformation of pure bipartite entangled states which we may frame as 'Incomparable states can not be deterministically transformed into one another by LOCC performed on the subsystems' [10, 11. The peculiarity of the existence of incomparable states is that it doesn't care about the amount of entanglement contained in the states. Though one of the two states has a greater entanglement content than the other, but the transformation can not be happen by deterministic LOCC for a pair of incomparable states. The existence of such class of states itself shows a peculiar feature of LOCC which can be studied to explore other areas of restrictions on the physical systems. Here we explore its connection with an impossible operation of quantum system.

Non-existence of universal exact flipping machine is a kind of constraint on the quantum systems that has been directly observed from unitary dynamics of the quantum evolution [3]. One could verify that the universal exact flipper, which if operated on an arbitrary state, will reverse the spin polarization direction, is not unitary but an anti-unitary operation and thus it is not a physical operation. No-flipping principle says that exact flipping of an unknown qubit state, is not possible. Like no-cloning, no-deleting principles, it is also a fundamental restriction to the allowable operations on a quantum system though this is an impossibility acting only on a single quantum system whereas we require at least two subsystems to describe nocloning, no-deleting principles. One interesting observation is found by Gisin and Popescu 3] and also by Massar [13] that the two-anti parallel-spin state $|\vec{n}, \overrightarrow{-n}\rangle$ contains more information than two-parallel-spin state $|\vec{n}, \vec{n}\rangle$. It is conjectured that the origin of this feature is the property that the anti parallel spin states span the whole four dimensional Hilbert space of two spin $\frac{1}{2}$ system, while the parallel spin $\frac{1}{2}$ system spans only a three dimensional subspace of the symmetric states. Then the optimal flipping machine is proposed by Buzek et.al. [12. The no-flipping principle has a completely different status from that of no-cloning, no-deleting principles as unlike those cases, there always exist a quantum machine which can act as an exact flip- 
per for any two non-orthogonal qubit states. Not only that, the largest set of states that can be flipped by a single flipper machine is any great circle of the Bloch sphere 14. It is also found that any three states of the Bloch sphere, not lying in one great circle can not be flipped by a single quantum machine. This no-flipping principle has already shown to be a consequence of no-signalling and non increase of entanglement by local operations [8].

Here we present a scheme that relates the impossibility of exact flipping of arbitrary qubit states with a seemingly different kind of impossibility of inter conversion of incomparable states by deterministic LOCC assuming the usual quantum mechanical formalism. We first show the impossibility of exact flipping for the three Bloch vectors along $x, y, z$, through incomparability. Then we show that by assuming the inter conversion of two incomparable states by deterministic LOCC one can generate a flipping machine for the whole Bloch sphere that reverses the directions of every Bloch vectors. Lastly we consider the first problem, the impossibility of universal exact flipping machine for any three qubits not lying on a great circle via incomparability. Now before going to describe our results, we first briefly mention incomparability criterion of a pair of pure bipartite entangled states.

The notion of incomparability of a pair of bipartite pure states is prescribed by M. A. Nielsen [10]. Suppose we want to convert the pure bipartite state $|\Psi\rangle$ to $|\Phi\rangle$ shared between two parties, say, Alice and Bob by deterministic LOCC. Consider $|\Psi\rangle,|\Phi\rangle$ in the Schmidt bases $\left\{\left|i_{A}\right\rangle,\left|i_{B}\right\rangle\right\}$ with decreasing order of Schmidt coefficients: $|\Psi\rangle=\sum_{i=1}^{d} \sqrt{\alpha_{i}}\left|i_{A} i_{B}\right\rangle,|\Phi\rangle=\sum_{i=1}^{d} \sqrt{\beta_{i}}\left|i_{A} i_{B}\right\rangle$, where $\alpha_{i} \geq \alpha_{i+1} \geq 0$ and $\beta_{i} \geq \beta_{i+1} \geq 0$, for $i=1,2, \cdots, d-1$, and $\sum_{i=1}^{d} \alpha_{i}=$ $1=\sum_{i=1}^{d} \beta_{i}$. And denote the Schmidt vectors as $\lambda_{\Psi} \equiv\left(\alpha_{1}, \alpha_{2}, \cdots, \alpha_{d}\right)$, $\lambda_{\Phi} \equiv\left(\beta_{1}, \beta_{2}, \cdots, \beta_{d}\right)$. Then Nielsen's criterion says $|\Psi\rangle \rightarrow|\Phi\rangle$ is possible with certainty under LOCC if and only if $\lambda_{\Psi}$ is majorized by $\lambda_{\Phi}$, denoted by $\lambda_{\Psi} \prec \lambda_{\Phi}$ and described as,

$$
\sum_{i=1}^{k} \alpha_{i} \leq \sum_{i=1}^{k} \beta_{i} \quad \forall \quad k=1,2, \cdots, d
$$

As a consequence of non-increase of entanglement by LOCC, if $|\Psi\rangle \rightarrow|\Phi\rangle$ is possible under LOCC with certainty, then $E(|\Psi\rangle) \geq E(|\Phi\rangle)$ [where $E(\cdot)$ denote the von-Neumann entropy of the reduced density operator of any subsystem and known as the entropy of entanglement]. If the above criterion (1) does not hold, then it is usually denoted by $|\Psi\rangle \not \rightarrow|\Phi\rangle$. Though it may happen that $|\Phi\rangle \rightarrow|\Psi\rangle$ under LOCC. Now if it happens that $|\Psi\rangle \not \rightarrow|\Phi\rangle$ and $|\Phi\rangle \not \rightarrow|\Psi\rangle$ then we denote it as $|\Psi\rangle \not \rightarrow|\Phi\rangle$ and describe $(|\Psi\rangle,|\Phi\rangle)$ as a pair of incomparable states. One of the interesting feature of such incomparable pairs is that we are unable to say that which state has a greater amount of entanglement content than the other. Also for $2 \times 2$ systems there are 
no pair of pure entangled states which are incomparable as described above. Now we explicitly mention the criterion of incomparability for a pair of pure entangled states $|\Psi\rangle,|\Phi\rangle$ of $m \times n$ system where $\min \{m, n\}=3$. Suppose the Schmidt vectors corresponding to the two states are $\left(a_{1}, a_{2}, a_{3}\right)$ and $\left(b_{1}, b_{2}, b_{3}\right)$ respectively, where $a_{1}>a_{2}>a_{3}, b_{1}>b_{2}>b_{3}, a_{1}+a_{2}+a_{3}=1=b_{1}+b_{2}+b_{3}$. Then it follows from Nielsen's criterion that $|\Psi\rangle,|\Phi\rangle$ are incomparable if and only if either of the two relations,

$$
\begin{aligned}
& a_{1}>b_{1} \text { and } b_{1}+b_{2}>a_{1}+a_{2} \\
& b_{1}>a_{1} \text { and } a_{1}+a_{2}>b_{1}+b_{2} \text { hold, } \\
& \text { or alternately, } \\
& a_{1}>b_{1}>b_{2}>a_{2}>a_{3}>b_{3} \\
& b_{1}>a_{1}>a_{2}>b_{2}>b_{3}>a_{3}
\end{aligned}
$$

hold.

At first to sense the idea of our work, consider three states representing the three axis of the Bloch sphere in the usual basis, as $\left|\psi_{x}\right\rangle=\frac{|0\rangle+|1\rangle}{\sqrt{2}},\left|\psi_{y}\right\rangle=$ $\frac{|0\rangle+i|1\rangle}{\sqrt{2}},\left|\psi_{z}\right\rangle=|0\rangle$. We choose the particular setting of a pure bipartite state in the form

$$
\left|\Psi^{i}\right\rangle_{A B}=\frac{1}{\sqrt{3}}\left\{|0\rangle_{A}\left|\psi_{z} \psi_{z}\right\rangle_{B}+|1\rangle_{A}\left|\psi_{x} \psi_{y}\right\rangle_{B}+|2\rangle_{A}\left|\psi_{y} \psi_{x}\right\rangle_{B}\right\}
$$

This is a three particle state, shared between two space-like separated parties Alice and Bob, so that Alice has a qutrit and Bob has two qubits, i.e., as a bipartite state it belongs to a $3 \times 4$ system. The reduced density matrix of Bob's subsystem admits a representation in terms of the three states $\left|\psi_{x}\right\rangle,\left|\psi_{y}\right\rangle,\left|\psi_{z}\right\rangle$. The Schmidt vector corresponding to the reduced system on Alice's side is $\lambda^{i}=\left(\frac{2}{3}, \frac{1}{6}, \frac{1}{6}\right)$. If the existence of exact flipping machine for the three states $\left|\psi_{x}\right\rangle,\left|\psi_{y}\right\rangle,\left|\psi_{z}\right\rangle$ is possible, then by applying this machine to one of the two particles on Bob's side, the joint state between them can be exactly transformed to the state

$$
\left|\Psi^{f}\right\rangle_{A B}=\frac{1}{\sqrt{3}}\left\{|0\rangle_{A}\left|\psi_{z} \overline{\psi_{z}}\right\rangle_{B}+e^{i \chi}|1\rangle_{A}\left|\psi_{x} \overline{\psi_{y}}\right\rangle_{B}+e^{i \eta}|2\rangle_{A}\left|\psi_{y} \overline{\psi_{x}}\right\rangle_{B}\right\}
$$

where $\chi, \eta$ are some arbitrary phases. The final reduced density matrix on Alice's side has the Schmidt vector $\lambda^{f}=\left(\frac{1}{3}+\frac{1}{2 \sqrt{3}}, \frac{1}{3}, \frac{1}{3}-\frac{1}{2 \sqrt{3}}\right)$. From equation (2) it is easy to check that $\left|\Psi^{i}\right\rangle_{A B},\left|\Psi^{f}\right\rangle_{A B}$ is a pair of incomparable states. Hence by Nielsen's criterion it is impossible to locally transform $\left|\Psi^{i}\right\rangle_{A B}$ to $\left|\Psi^{f}\right\rangle_{A B}$ with certainty. In this example we see that the impossibility of an operation in quantum mechanics can be established from the contradiction that it forces two incomparable states to become comparable by LOCC with certainty. 
The result shows, how a local anti-unitary operation evolves the system in an unphysical way. To explore this unphysical nature of anti-linear operators, we search for a joint system between some separated parties, as on a single system it is really difficult to distinguish unitary and anti-unitary operators. Here we observe that applying an anti-unitary operator (say, $L$ ) locally on a joint system of $3 \times(2 \times 2)$ dimension, i.e., applying the operator $I_{A} \otimes(I \otimes L)_{B}$ on the joint system, there arises a case of incomparability. The bi-partite entanglement of the joint system changes and it changes in such a manner that there is no way to compare the initial and final joint states locally. Now one can ask whether the converse is also to be happen or not, i.e., is it possible to generate an exact flipping machine by assuming inter conversion of at least a pair of incomparable states by deterministic LOCC? Our next example is going to explore it where by exact flipping we mean a machine that can reverse the direction of any Bloch vector. This machine usually generalizes the notion of flipping from any pure qubit to any state on the whole Bloch sphere.

Consider a pair of pure entangled states, shared between Alice and Bob situated in two distant places, in the form

$$
\begin{aligned}
& |\Psi\rangle_{A B}=\sqrt{.51}|0\rangle_{A}|0\rangle_{B}+\sqrt{.30}|1\rangle_{A}|1\rangle_{B}+\sqrt{.19}|2\rangle_{A}|2\rangle_{B} \\
& |\Phi\rangle_{A B}=\sqrt{.49}|0\rangle_{A}|0\rangle_{B}+\sqrt{.36}|1\rangle_{A}|1\rangle_{B}+\sqrt{.15}|2\rangle_{A}|2\rangle_{B}
\end{aligned}
$$

The Schmidt vectors corresponding to one of the subsystems of $|\Psi\rangle_{A B},|\Phi\rangle_{A B}$ are $\lambda^{\Psi}=(.51, .30, .19)$ and $\lambda^{\Phi}=(.49, .36, .15)$. Using equation (2) we find, $\left(|\Psi\rangle_{A B},|\Phi\rangle_{A B}\right)$ are a pair of incomparable states. Suppose Bob has a two qubit system on his side and the orthogonal states $\left\{|0\rangle_{B},|1\rangle_{B},|2\rangle_{B}\right\}$ have the form,

$$
|0\rangle_{B}=|\psi\rangle_{B_{1}}|\psi\rangle_{B_{2}},|1\rangle_{B}=|\bar{\psi}\rangle_{B_{1}}|\psi\rangle_{B_{2}},|2\rangle_{B}=|\bar{\psi}\rangle_{B_{1}}|\bar{\psi}\rangle_{B_{2}}
$$
where $|\psi\rangle$ is an arbitrary qubit state with Bloch vector $\overrightarrow{n_{\psi}}$, i.e., $|\psi\rangle\langle\psi|=$ $\frac{1}{2}\left[I+\overrightarrow{n_{\psi}} \cdot \vec{\sigma}\right]$ and $|\bar{\psi}\rangle$ is orthogonal to $|\psi\rangle$.

Now tracing out Alice's system and the second qubit of Bob's side (i.e., system $B_{2}$ ), we get one qubit reduced subsystem of Bob corresponding to the two joint systems in (5) as,

$$
\begin{aligned}
& \rho^{\Psi}=\operatorname{Tr}_{A B_{2}}\{|\Psi\rangle\langle\Psi|\}=\frac{1}{2}\left[I+.02 \overrightarrow{n_{\psi}} \cdot \vec{\sigma}\right] \\
& \rho^{\Phi}=\operatorname{Tr}_{A B_{2}}\{|\Phi\rangle\langle\Phi|\}=\frac{1}{2}\left[I-.02 \overrightarrow{n_{\psi}} \cdot \vec{\sigma}\right] .
\end{aligned}
$$

If by any local operation it is possible to transform the joint state between Alice and Bob from $|\Psi\rangle$ to $|\Phi\rangle$, then the reduced state of one qubit subsystem on Bob's side will be changed accordingly from $\rho^{\Psi}$ to $\rho^{\Phi}$ exactly, by LOCC. It is clear that the spin direction $\overrightarrow{n_{\psi}}$ of the arbitrary qubit state $|\psi\rangle$ is reversed after the operation, i.e., transformed to $-\overrightarrow{n_{\psi}}$. So, if we extend the LOCC 
transformation criterion so that the states $|\Psi\rangle,|\Phi\rangle$ are interconvertible by some operation then consequently on a subsystem, the spin-polarization of an arbitrary qubit state is being reversed. This is quite similar of preparing an arbitrary spin flipper machine and is an alternative way of establishing the incomparable nature of the pair of states in equation (5).

This two examples in contrast of each other show, how an impossible local operation is connected with the restrictions imposed on state transformation by LOCC. The root of this connection is the anti-unitary nature of the exact universal spin-flipping operation. In the case of state transformation criterion, the allowed local operations on each parties are such that as a whole it can be implemented by an unitary evolution and if we restrict further individual local operations as unitary then bipartite entanglement cannot be changed under such local unitary operations. We, however consider a local operation which is anti-unitary and it is observed that anti-unitary operator acts in a nonphysical way. The above two examples show that it is not possible to extend the class of physical operations so as to incorporate anti-unitary operations [15].

To generalize the main result corresponding to the first example, we consider three arbitrary states not lying in one great circle in their simplest form, $|0\rangle,|\psi\rangle=a|0\rangle+b|1\rangle,|\phi\rangle=c|0\rangle+d e^{i \theta}|1\rangle$ where a, b, c, d are real numbers satisfying the relation $a^{2}+b^{2}=1=c^{2}+d^{2}$ and $0<\theta<\pi$. Suppose two spatially separated parties Alice and Bob are sharing the entangled state

$$
|\Omega\rangle_{A B}=\frac{1}{\sqrt{3}}\left\{|0\rangle_{A}|00\rangle_{B}+|1\rangle_{A}|\psi \phi\rangle_{B}+|2\rangle_{A}|\phi \psi\rangle_{B}\right\}
$$

where Alice has a 3-dimensional orthogonal local system, having the basis, $\{|0\rangle,|1\rangle,|2\rangle\}$ and Bob has a two qubit system. The reduced density matrix of Alice's side is

$$
\begin{aligned}
\rho_{A}^{i}=\frac{1}{3}\{P[|0\rangle]+P[|1\rangle]+P[|2\rangle]+a c(|0\rangle\langle 1|+| 1\rangle\langle 0|+| 0\rangle\langle 2| \\
\left.+|2\rangle\langle 0|)+|\langle\psi \mid \phi\rangle|^{2}(|1\rangle\langle 2|+| 2\rangle\langle 1|)\right\}
\end{aligned}
$$

Consider that Bob has another system which acts as a exact flipping machine defined on these three states $|0\rangle,|\psi\rangle,|\phi\rangle$. The flipping operation can be described as

$$
\begin{aligned}
& |0\rangle \longrightarrow|1\rangle \\
& |\psi\rangle \longrightarrow e^{i \mu}|\bar{\psi}\rangle \\
& |\phi\rangle \longrightarrow e^{i \nu}|\bar{\phi}\rangle
\end{aligned}
$$

where $|\bar{\psi}\rangle,|\bar{\phi}\rangle$ are the states orthogonal to $|\psi\rangle,|\phi\rangle$ respectively and $\mu$ and $\nu$ are some arbitrary phases. Now assume that Bob applies the flipping machine on any one of his two particles (say, on the second particle). After 
this local operation on Bob's subsystem, the shared state between Alice and Bob takes the form,

$$
|\Omega\rangle_{A B}^{f}=\frac{1}{\sqrt{3}}\left\{|0\rangle_{A}|01\rangle_{B}+e^{i \nu}|1\rangle_{A}|\psi \bar{\phi}\rangle_{B}+e^{i \mu}|2\rangle_{A}|\phi \bar{\psi}\rangle_{B}\right\}
$$

The final density matrix of Alice's side is

$$
\begin{aligned}
\rho_{A}^{f}= & \frac{1}{3}\{P[|0\rangle]+P[|1\rangle]+P[|2\rangle] \\
& -a c\left(e^{-i \nu}|0\rangle\left\langle 1\left|+e^{i \nu}\right| 1\right\rangle\left\langle 0\left|+e^{-i \mu}\right| 0\right\rangle\left\langle 2\left|+e^{i \mu}\right| 2\right\rangle\langle 0|\right) \\
& \left.+(\langle\phi \mid \psi\rangle)^{2} e^{i(\nu-\mu)}|1\rangle\left\langle 2\left|+(\langle\psi \mid \phi\rangle)^{2} e^{i(\mu-\nu)}\right| 2\right\rangle\langle 1|\right\}
\end{aligned}
$$

The eigenvalue equation on the initial local density matrix $\rho_{A}^{i}$ is,

$$
(1-3 \lambda)^{3}-3(1-3 \lambda) A+B=0
$$

and that of the final local density matrix $\rho_{A}^{f}$ is,

$$
(1-3 \lambda)^{3}-3(1-3 \lambda) A+B^{\prime}=0
$$

where $A=\frac{1}{3}\left[2 a^{2} c^{2}+|\langle\psi \mid \phi\rangle|^{4}\right], B=2 a^{2} c^{2}|\langle\psi \mid \phi\rangle|^{2}$ and $B^{\prime}=2 a^{2} c^{2} \operatorname{Re}\left\{\langle\phi \mid \psi\rangle^{2}\right\}$. It is to be noticed that the phase factors $e^{i \mu}, e^{i \nu}$ vanishes from this stage. So the result obtained doesn't care the phase factor of the operation. The roots of the equation (12) are $\alpha_{1}=\frac{1}{3}\left\{1-2 \sqrt{A} \cos \left(\frac{2 \pi}{3}+\theta^{i}\right)\right\}, \alpha_{2}=\frac{1}{3}\left\{1-2 \sqrt{A} \cos \theta^{i}\right\}$ and $\alpha_{3}=\frac{1}{3}\left\{1-2 \sqrt{A} \cos \left(\frac{2 \pi}{3}-\theta^{i}\right)\right\}$ and the roots of the equation (13) are $\beta_{1}=\frac{1}{3}\left\{1-2 \sqrt{A} \cos \left(\frac{2 \pi}{3}+\theta^{f}\right)\right\}, \beta_{2}=\frac{1}{3}\left\{1-2 \sqrt{A} \cos \theta^{f}\right\}$ and $\beta_{3}=\frac{1}{3}\{1-$ $\left.2 \sqrt{A} \cos \left(\frac{2 \pi}{3}-\theta^{f}\right)\right\}$ where, $\cos 3 \theta^{i}=\frac{-B}{2 \sqrt{A^{3}}}$, and $\cos 3 \theta^{f}=\frac{-B^{\prime}}{2 \sqrt{A^{3}}}$. Now to compare the states $|\Omega\rangle_{A B}$ to $|\Omega\rangle_{A B}^{f}$ we do not need the explicit values of the Schmidt coefficients of the corresponding reduced density matrices but only we have to find the relation between them.

Here $B=B^{\prime}+4 a^{2} b^{2} c^{2} d^{2} \sin ^{2} \theta$, so $B \geq 0, B \geq B^{\prime}$. If, $0<B^{\prime}<B$, then we have $0>\cos \left(3 \theta^{f}\right)>\cos \left(3 \theta^{i}\right)$, this will imply that $3 \theta^{i}, 3 \theta^{f} \in\left(\frac{\pi}{2}, \frac{3 \pi}{2}\right)$. We find four subcases corresponding to the different regions of $\theta^{i}, \theta^{f}$. Firstly we consider the case when $3 \theta^{i}, 3 \theta^{f} \in\left(\frac{\pi}{2}, \pi\right)$. In this region, $\cos \left(3 \theta^{f}\right)>\cos \left(3 \theta^{i}\right) \Rightarrow$ $3 \theta^{i}>3 \theta^{f}$. So, we have $\frac{\pi}{6}<\theta^{f}<\theta^{i}<\frac{\pi}{3}$, which imply $\frac{\sqrt{3}}{2}>\cos \left(\theta^{f}\right)>$ $\cos \left(\theta^{i}\right)>\frac{1}{2}$. Then $\alpha_{2}, \beta_{2}$ will lie on the region, $\frac{1}{3}(1-\sqrt{A})>\alpha_{2}>\beta_{2}>\frac{1}{3}(1-$ $\sqrt{3 A})$. Proceeding in this way, we find $\frac{1}{3}(1+2 \sqrt{A})>\alpha_{1}>\beta_{1}>\frac{1}{3}(1+\sqrt{3 A})$ and $\frac{1}{3}>\beta_{3}>\alpha_{3}>\frac{1}{3}(1-\sqrt{A})$. Thus the eigenvalues of $\rho_{A}^{i}, \rho_{A}^{f}$ are related as $\alpha_{1}>\beta_{1}>\beta_{3}>\alpha_{3}>\alpha_{2}>\beta_{2}$. So by equation (2) the states $|\Omega\rangle_{A B},|\Omega\rangle_{A B}^{f}$ are incomparable in this region.

In a similar manner we investigated the other regions (for cases $B^{\prime}>0$ and $B^{\prime}<0$ ) which also show incomparability between the two bipartite states (See Appendix). 
Equations (12) and (13) will be identical (and hence the Schmidt vectors of $\rho_{A}^{i}$ and $\rho_{A}^{f}$ ) when $B=B^{\prime}$, which imply abcd $\sin \theta=0$, i.e., the three states $|0\rangle,|\psi\rangle,|\phi\rangle$, on which the flipping machine is defined, will lie on one great circle of the Bloch sphere. This is clear from the fact that there exists exact flipping machine for the set of states taken from one great circle [8, 14].

Thus, if the exact flipping machine does exist, and is applied locally on one subsystem of the initial pure bipartite state, then an impossible transformation is shown to occur. Obviously this impossibility comes through our assumption on the existence of universal exact flipping machine. It is interesting to observe that the arbitrary phase factor of the flipping operation we have considered, does not make a difference in the result obtained.

This work shows an interplay between the notion of incomparability and no-flipping principle. It indicates no-flipping can be used to determine the interrelations between LOCC and entanglement behavior of the quantum system. We observe, the incomparability criterion of local state transformations is also capable of revealing some more fundamental properties of the quantum systems. It can detect operations which are nonphysical in nature such as, here it is anti-unitary. Naturally one could conjecture that the two impossibilities are equivalent, as they both require anti-unitary operators. Our results support this conjecture. It also exhibit the impossibility of extending LOCC operations to incorporate anti-unitary operators which can create an increase of information content of the system, as anti-parallel spin states contain more information than that of the parallel ones.

Acknowledgement. The authors thank the referees for their valuable comments and suggestions. The authors also thank G. Kar for useful discussions in preparation of this paper. I.C. acknowledges CSIR, India for providing fellowship during this work.

\section{References}

[1] W. K. Wootters and W. H. Zurek, Nature 299, 802 (1982).

[2] A. K. Pati and S. L. Braunstein, Nature 404, 164 (2000); W. H. Zurek, Nature 404, 130 (2000) .

[3] N. Gisin and S. Popescu, Phys. Rev. Lett. 83, 432-435 (1999).

[4] A. K. Pati, Phys. Rev. A 66, 062319 (2002).

[5] D. Dieks, Phys. Lett. A 92, 271 (1982); H. P. Yuen, Phys. Lett. A 113, 405 (1986). 
[6] A. K. Ekert, Phys. Rev. Lett. 67, 661 (1991); C. H. Bennett and S. J. Wiesner, Phys. Rev. Lett. 69, 2881 (1992); C. H. Bennett, G. Brassard, C. Crépeau, R. Jozsa, A. Peres and W. K. Wootters, Phys. Rev. Lett. 70, 1895 (1993).

[7] N. Gisin, Phys. Lett. A 242, 1-3 (1998); A. K. Pati and S. L. Braunstein, Phys. Lett. A 315, 208-212 (2003); A. K. Pati, Phys. Lett. A 270, 103 (2000).

[8] I. Chattopadhyay, S. K. Choudhary, G. Kar, S. Kunkri and D. Sarkar, Phys. Lett. A 351, 384-387 (2006).

[9] M. Horodecki, R. Horodecki, A. Sen(De) and U. Sen, arXiv: quant-ph/0306044.

[10] M. A. Nielsen, Phys. Rev. Lett. 83, 436 (1999); M. A. Nielsen and I. L. Chuang , Quantum Computation and Quantum Information' (Cambridge University Press, 2000).

[11] I. Chattopadhyay and D. Sarkar, Quantum Information and Computation, 5, 247-257 (2005).

[12] V. Buzek, M. Hillery and R. F. Werner, Phys. Rev. A 60, R2626-R2629 (1999); S. J. van Enk, Phys. Rev. Lett. 95, 010502 (2005).

[13] S. Massar, Phys. Rev. A 62, 040101(R) (2000).

[14] S. Ghosh, A. Roy and U. Sen, Phys. Rev. A 63, 014301 (2000); A. K. Pati, Phys. Rev. A 63, 014302 (2001).

[15] C. Simon, V. Buzek and N. Gisin, Phys. Rev. Lett. 87, 170405 (2001).

\section{Appendix}

For $0<B^{\prime}<B$, we have the other three possible regions as follows. When both of $3 \theta^{i}, 3 \theta^{f} \in\left(\pi, \frac{3 \pi}{2}\right)$ then, $\alpha_{1}>\beta_{1}>\beta_{2}>\alpha_{2}>\alpha_{3}>\beta_{3}$. When, $3 \theta^{i} \in\left(\frac{\pi}{2}, \pi\right)$ and $3 \theta^{f} \in\left(\pi, \frac{3 \pi}{2}\right)$ then, $\alpha_{1}>\beta_{1}>\beta_{2}>\alpha_{3}>\alpha_{2}>\beta_{3}$. When, $3 \theta^{i} \in\left(\pi, \frac{3 \pi}{2}\right)$ and $3 \theta^{f} \in\left(\frac{\pi}{2}, \pi\right)$ then, $\alpha_{1}>\beta_{1}>\beta_{3}>\alpha_{2}>\alpha_{3}>\beta_{2}$.

Otherwise, $B^{\prime}<0<B$, then we have $\cos \left(3 \theta^{f}\right)>0>\cos \left(3 \theta^{i}\right)$, which implies $3 \theta^{i} \in\left(\frac{\pi}{2}, \frac{3 \pi}{2}\right)$ and $3 \theta^{f} \in\left\{\left(0, \frac{\pi}{2}\right) \cup\left(\frac{3 \pi}{2}, 2 \pi\right)\right\}$. The following subcases for different regions of $\theta^{i}, \theta^{f}$ are considered separately.

When, $3 \theta^{i} \in\left(\frac{\pi}{2}, \pi\right)$ and $3 \theta^{f} \in\left(0, \frac{\pi}{2}\right)$ then, $\alpha_{1}>\beta_{1}>\beta_{3}>\alpha_{3}>\alpha_{2}>\beta_{2}$. When, $3 \theta^{i} \in\left(\frac{\pi}{2}, \pi\right)$ and $3 \theta^{f} \in\left(\frac{3 \pi}{2}, 2 \pi\right)$ then, $\alpha_{1}>\beta_{1}>\beta_{2}>\alpha_{3}>\alpha_{2}>\beta_{3}$. When, $3 \theta^{i} \in\left(\pi, \frac{3 \pi}{2}\right)$ and $3 \theta^{f} \in\left(0, \frac{\pi}{2}\right)$ then, $\alpha_{1}>\beta_{1}>\beta_{3}>\alpha_{2}>\alpha_{3}>\beta_{2}$. When, $3 \theta^{i} \in\left(\pi, \frac{3 \pi}{2}\right)$ and $3 \theta^{f} \in\left(\frac{3 \pi}{2}, 2 \pi\right)$ then, $\alpha_{1}>\beta_{1}>\beta_{2}>\alpha_{2}>\alpha_{3}>\beta_{3}$. In all the above cases by equation (2) the states are incomparable. 